\title{
A CONTINUOUS FUNCTION WITH TWO CRITICAL POINTS
}

\author{
BY NICOLAAS H. KUIPER ${ }^{1}$ \\ Communicated by R. P. Boas, February 6, 1961
}

A real $C^{s}$-function $f: X \rightarrow \mathbf{R}$ on an $n$-dimensional $C^{s}$-manifold with $s \geqq 0$, is called $C^{s}$-nondegenerate $C^{s}$-ordinary at a point $p \in X$, in case a system of $n C^{s}$-coordinates ( $C^{s}$-functions) $\phi_{1}, \cdots, \phi_{n}$ exists, which defines a $C^{s}$-diffeomorphism $\kappa$ of some neighborhood $V(p)$ of $p$ into $\mathbf{R}^{n}$, and such that for some constant $\lambda_{p}>0$

$$
\text { (1) } \phi_{i}(p)=0, i=1, \cdots, n ; \phi_{n}(q)=\lambda_{p}\{f(q)-f(p)\}
$$

for $q \in V(p) \subset X$.

If $C^{s}$-coordinates and $\lambda_{p}>0$ exist such that

$$
\begin{aligned}
\phi_{i}(p) & =0, \quad i=1, \cdots, n ; \\
-\sum_{1}^{r} \phi_{i}^{2}(q)+\sum_{r+1}^{n} \phi_{j}^{2}(q) & =\lambda_{p}\{f(q)-f(p)\}
\end{aligned}
$$

then the function is called $C^{s}$-critical of index $r$ and $C^{s}$-nondegenerate at $p$.

A function which is $C^{s}$-nondegenerate at every point $p \in X$ is called a $C^{*}$-nondegenerate function.

We will restrict our considerations to the topological case $s=0$ of continuous functions on topological manifolds and we will omit $C^{0}$ from the notation in the sequel. By function we will mean continuous function, etc.

A compact manifold without boundary is called a closed manifold. A nondegenerate function on a closed manifold has at least one critical point $p_{1}$ of index $n$ and one critical point $p_{0}$ of index 0 , corresponding respectively with the maximum and the minimum of the function. We prove the

THEOREM. If $X$ is a closed $n$-dimensional manifold and $f: X \rightarrow \mathbf{R} a$ continuous nondegenerate function with exactly two critical points, then $X$ is homeomorphic to the $n$-sphere $S^{n}{ }^{2}$

1 The author has a research grant from the National Science Foundation, NSFG-13989.

2 Reeb [2] proved the corresponding theorem for the differentiable case. Morse [1] proved that $X$ is a homotopy-sphere, and he also has a proof of the theorem we present (unpublished as yet). 
Proof. A. The local droppings $T_{p}$. We place ourselves in the assumptions of the theorem and we call the function $f$ "height." We consider a coordinate system for every point $p \in X$, obeying (1) or (2), but for which moreover the image $\kappa_{p}(V(p)) \subset \mathbf{R}^{n}$ is the open $n$ ball

$$
r<5 \text {, }
$$

where the "polar coordinates" $r$ (radius) and $\omega$ (unit vector) are defined by

$$
r=\left(\sum_{j}^{n} \phi_{j}^{2}\right)^{1 / 2}, \quad \omega=\left(\phi_{1} / r, \phi_{2} / r, \cdots, \phi_{n} / r\right) .
$$

For any such coordinate system $\kappa: V(p) \rightarrow \boldsymbol{R}^{n}$ we also define the open set

$$
U_{t}(p)=\{q \mid q \in V(p) \subset X, r(q)<t\} .
$$

Next we define a homeomorphism $T_{p}$ for every $p \in X$. If $p$ is an ordinary point then we proceed as follows:

Let $h(t)$ be a real $C^{\infty}$-function with the properties

$$
\begin{aligned}
& h(t) \begin{cases}=0, & |t| \geqq 4, \\
>0, & |t|<4, \\
=h(0), & |t| \leqq 1,\end{cases} \\
&\left|h^{\prime}(t)\right|<1 / 2, \text { any } t .
\end{aligned}
$$

The homeomorphism $T_{p}$ is given by:

$$
\begin{aligned}
\phi_{i}\left(T_{p}(q)\right) & =\phi_{i}(q), \quad i=1, \cdots, n-1 \\
\phi_{n}\left(T_{p}(q)\right) & =\phi_{n}(q)-h(r(q)) \\
T_{p}(q) & =q,
\end{aligned} \quad \begin{aligned}
q & \in V(p), \\
q & \notin V(p) .
\end{aligned}
$$

As the Jacobian of the corresponding $C^{\infty}$-transformation of the coordinates for $q \in V(p)$ does not vanish, and $T(q)=q$ for $q \notin U_{4}(p)$, it follows that $T_{p}$ is a global homeomorphism of $X$. Observe that the continuous function

$$
q \rightarrow f\left(T_{p}(q)\right)-f(q): X \rightarrow \mathbf{R}
$$

takes the value zero for $q \notin U_{4}(p)$ and is negative for $q \in U_{4}(p)$. It takes a negative maximal value on the set $\overline{U_{3}(p)}$, the closure in $X$ of $U_{3}(p)$. Under $T_{p}$ no point is mapped into a higher level of $f$, and every point of $U_{4}(p)$ is mapped into a lower level. 
If $p$ is a critical point of index $n$ we use a real $C^{\infty}$-function $k(t)$ with the properties

$$
\begin{aligned}
& k(t) \begin{cases}=t, & \text { for } t \geqq 4, \\
=2 t, & 0 \leqq t \leqq 1, \\
>t, & 0 \leqq t<4,\end{cases} \\
& k^{\prime}(t)>0, \quad t \geqq 0 .
\end{aligned}
$$

The homeomorphism $T_{p}$ is now defined in terms of polar coordinates (4) by:

$$
\begin{aligned}
& \left.\begin{array}{l}
\omega\left(T_{p}(q)\right)=\omega(q) \\
r\left(T_{p}(q)\right)=k(r(q))
\end{array}\right\} \quad \text { for } q \in V(p), \\
& T_{p}(q)=q \quad \text { for } q \notin V(p) .
\end{aligned}
$$

The restriction of $T_{p}$ to $U_{1}(p)$ is represented by a geometrical multiplication with factor 2 in coordinate space.

The point $p$ and every point $q \notin U_{4}(p)$ is invariant under $T_{p}$. Every other point in $X$ is mapped into a lower level.

In the case of critical point of index zero we use the function $k^{-1}$, the inverse of $k$, and proceed analogously.

B. The global dropping $T$. Under the given assumptions there is a critical point $p_{1}$ of index $n$ (maximum), a critical point $p_{0}$ of index 0 (minimum), and no other critical point. Choose a finite number of coordinate systems $\kappa_{p_{i}}$ and homeomorphisms $T_{p_{i}}, i=0, \cdots, L$, of the kinds mentioned above, such that:

$$
\bigcup_{i=0}^{L} U_{3}\left(p_{i}\right)=X
$$

but

$$
\bigcup_{i=2}^{L} U_{4}\left(p_{i}\right) \cap\left[U_{2}\left(p_{0}\right) \cup U_{2}\left(p_{1}\right)\right]=\varnothing \text { (void). }
$$

(Compare the use of a partition of unity.) Let

$$
T=T_{p_{L}} T_{p_{L-1}} \cdots T_{p_{2}} T_{p_{1}} T_{p_{0}} .
$$

Then $T: X \rightarrow X$ is a global homeomorphism with exactly two invariant points, namely $p_{0}$ and $p_{1}$, which maps every other point into a lower level:

(12) $T\left(p_{0}\right)=p_{0} ; \quad T\left(p_{1}\right)=p_{1} ; f(T(q)) \leqq f(q)$ for $q \in X-p_{0}-p_{1}$. 
As the set $W_{\epsilon}=X-U_{2}\left(p_{1}\right)-U_{\epsilon}\left(p_{0}\right)$ for $0<\epsilon<1$, is compact, the nonnegative function

$$
f(q)-f(T(q))
$$

has a minimal value for $q \in W_{\epsilon}$ and this minimal value is positive. Call it $\delta_{\epsilon}=U$ and let $N_{\epsilon}$ be an integer such that

$$
N_{\epsilon} \delta_{\epsilon}>f\left(p_{1}\right)-f\left(p_{0}\right) \text {. }
$$

If we apply powers with consecutive exponents of the homeomorphism $T$, to any point $q \in W_{\mathrm{\epsilon}}$, then for some exponent $N \leqq N_{\mathrm{G}}$ we will find

$$
T^{N}(q) \in U_{\epsilon}\left(p_{0}\right)
$$

because with each new application of $T$ to the result obtained in the last step, we obtain a new point which is at a level at least $\delta_{e}$ lower, and after $N_{\epsilon}$ steps the point would have dropped totally more than the total range of the function $f$ over $X$. On the other hand, once the resulting point is in $U_{\epsilon}\left(p_{0}\right)$ any further application of $T$ will give a new point also in $U_{\epsilon}\left(p_{0}\right)$, because $T$ acts in $U_{\epsilon}\left(p_{0}\right)$ as a geometrical multiplication with factor $1 / 2$. Consequently

$$
T^{N} \cdot\left(X-U_{2}\left(p_{1}\right)\right) \subset U_{\epsilon}\left(p_{0}\right)
$$

and taking complements

$$
T^{N_{e}}\left(U_{2}\left(p_{1}\right)\right) \supset X-U_{e}\left(p_{0}\right) .
$$

Thus $X$ is covered by two discs:

$$
T^{N} \epsilon\left(U_{2}\left(p_{1}\right)\right) \cup U_{\epsilon}\left(p_{0}\right)=X
$$

and our theorem can be considered as a consequence of a theorem of Morton Brown. However, we like to present a complete explicit proof:

C. The homeomorphism $X \rightarrow S^{n}$.

As (14) holds for any $0<\epsilon<1$, it follows that for any $q \neq p_{0}$ there exists a smallest number $N_{q}$ such that

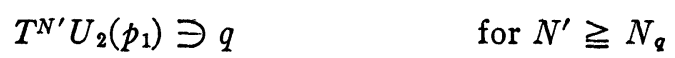

or

$$
T^{-N^{\prime}}(q) \in U_{2}\left(p_{1}\right) .
$$

Let $\kappa_{1}: U_{2}\left(p_{1}\right) \rightarrow \mathbf{R}^{n}$ be the restriction of the coordinate system at the critical point $p_{1}$ to the open set $U_{2}\left(p_{1}\right)$. Observe that for any $q \in U_{2}\left(p_{1}\right)$ :

$$
2^{k} \cdot \kappa_{1}\left[T^{-k}(q)\right]=\kappa_{1}(q), \quad k \geqq 0 .
$$


If $N^{\prime} \geqq N=N_{q}$ then in view of (17) we have in the vector space $\mathbf{R}^{n}$ :

$$
2^{N^{\prime}{ }^{\prime}}\left[T^{-N^{\prime}}(q)=2^{N} \cdot 2^{N^{\prime}-N} k_{1}\left(T^{-N^{\prime}+N} T^{-N} q\right)=2^{N} \kappa_{1}\left(T^{-N} q\right) .\right.
$$

Hence there exists a mapping $\kappa:\left(X-p_{0}\right) \rightarrow \mathbf{R}^{n}$ well defined by:

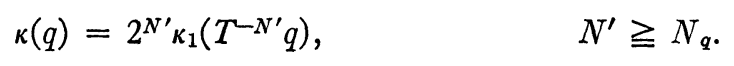

$\kappa$ is clearly locally a homeomorphism. $\kappa$ is onto the set $\bigcup_{j=0}^{\infty} 2^{2} \kappa_{1}\left(U_{2}\left(p_{1}\right)\right)$ $=\mathbf{R}^{n}$. If $q_{1}$ and $q_{2} \neq q_{1}$ are both different from $p_{0}$ then, for $N^{\prime} \geqq N_{q_{1}}+N_{\mathbf{q}_{2}}$,

$$
T^{-N^{\prime}}\left(q_{1}\right) \neq T^{-N^{\prime}}\left(q_{2}\right)
$$

and consequently $\kappa\left(q_{1}\right) \neq \kappa\left(q_{2}\right)$. So $\kappa:\left(X-p_{0}\right) \rightarrow \mathbf{R}^{n}$ is a homeomorphism and $X$ is homeomorphic to the one point compactification of $\mathbf{R}^{n}$, that is $S^{n}$.

\section{BIBLIOGRAPHY}

1. Marston Morse, Topologically non-degenerate functions on a compact $n$-manifold $M$, J. Analyse Math. vol. 7 (1959) pp. 189-208.

2. G. Reeb, Sur certaines propriêtés topologiques des variêtés feuilletées, Actualités Sci. Ind. No. 1183 (1952) pp. 91-154.

LANDBOUWHOGESCHOOL, WAGENINGEN, NETHERLANDS AND NORTHWESTERN UNIVERSITY 